\title{
Diagnosis of alzheimer disease through detection of eyes retinal blood vessels
}

\begin{abstract}
Today, Alzheimer's disease is rapidly growing and spreading. The disease is caused by the accumulation of Amyloid plaque deposits, thereby destroying the connection between nerve cells and the formation of neurofibrillary nods. According to recent research, one of the ways to diagnose Alzheimer's disease is by reflecting light into the patient's retinal blood vessels and using a machine vision system. Therefore, in this study, we have attempted to introduce a method to detect human retinal blood vessels to improve the disease. In the above method, using DRIVE bank images, they are first clustered and after applying the Unsharp filter and Radon conversion, the vessel is diagnosed so that early detection of Alzheimer's disease by counting the number of vessels present.
\end{abstract}

Keywords: alzheimer's disease, segmentation, eyes retinal blood vessels, radon conversion
Volume 4 Issue 2 - 2020

\author{
Shahed Salehzehi,' Elyas Irankhah² \\ 'Department of Biomedical Engineering, Islamic Azad university, \\ Iran \\ ${ }^{2}$ Department of Biomedical Engineering, International University \\ of Imam Reza (AS), Iran
}

\begin{abstract}
Correspondence: Shahed Salehzehi, Department of Biomedical Engineering, Islamic Azad university, Iran, Tel +989302681900, Email Shahed.salehzehi@gmail.com
\end{abstract}

Received: January 22, 2020 | Published: April 28, 2020

\section{Introduction}

Alzheimer's disease is a brain disorder and that gradually, in an irreversible way, destroys memory and thinking skills, and ultimately destroy the ability to perform the simplest of tasks. The pathology of the disease is known to occur in the brain cells of Alzheimer's patients with protein deposits called Amyloid plaques. These plaques not only block the communication between the nerve cells but also destroy the surrounding cells, resulting in neurofibrillary nods in the brain's nerve cells. Growth of this compound destroys brain cells and decreases the levels of some chemicals that transmit message between nerve cells and also destroy the communication between cells. Diagnosis is usually done through tests such as CT Scans, MRI, tapes and spinal fluid tests or psychiatric tests of memory and eye tracking tests. Symptoms usually appear in people who are in their mid-sixties, but early patterns may occur between the ages of 30 and 60 . Which is extremely rare. Depression is one of the early symptoms of Alzheimer's disease. About 50 percent of Alzheimer's patients are said to be depressed before they lose their memory. At present, the treatment process means more to prevent the progression of the disease and to control the symptoms and psychiatric-behavioral disorders that result format it. ${ }^{1-3}$ Given the Alzheimer's disease prevention and treatment processes, the detection of this disease through the human retinal blood vessels is a new achievement and could be a leap in the process of complete recovery. Since various methods have been used to identify blood vessels, this study has tried to use the simplest and most efficient method.

\section{Method and materials}

\section{Data}

The images used in this study included 20 images of healthy individuals selected from the DRIVE bank. The images above are $584 \times 565$. The proposed algorithm has been implemented and tested in MATLAB software.

\section{Segmentation}

Image segmentation is the process of segmenting a digital image into several parts. The purpose is to segment, simplify, or change the representation of an image to something that is both meaningful and easier to analyze. This method is usually used to find the location of objects and boundaries in the image. More precisely, it refers to a process in which each pixel is assigned a label so that the same labeled pixels have similar properties (Figure 1).,5

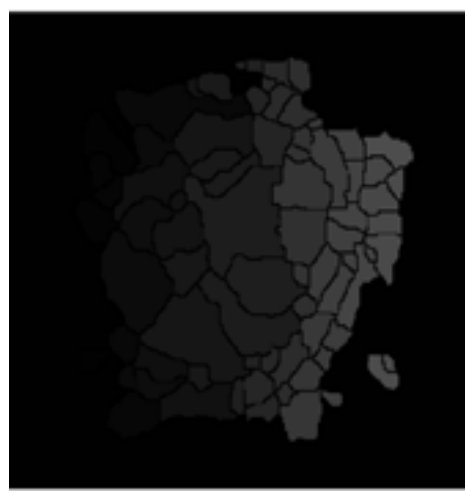

Figure I Retinal blood vessels segmentation.

\section{Unsharp filter}

This method, known as non-transparent masking, is a technique for sharpening images that is often available in digital image processing software, including MATLAB. This technique uses a single, uneven or unique image to create a mask of the original image. This filter is then combined with the original (positive) image to create a smaller image than the original. The resulting image, though clearer, may be a more accurate representation of the subject of the image (Figure 2).
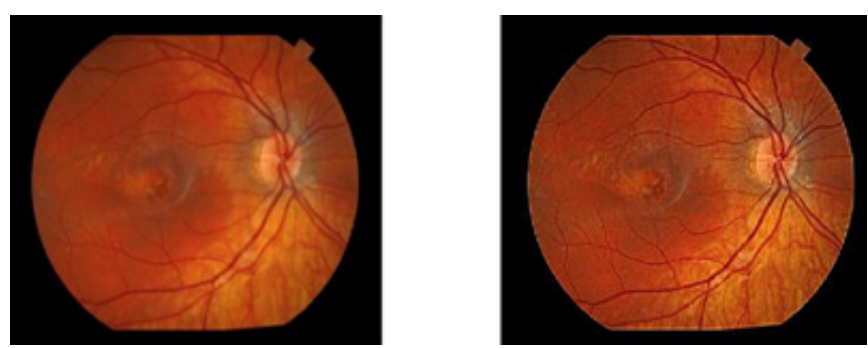

Figure 2 Sharpen of the retinal blood vessels in the Unsharp filter. 


\section{Radom conversion and vessel detection}

By creating an initial image, a good contrast is created between the artery and the background. At the same time, to improve the accuracy of the algorithm after image segmentation in each window the image contrast is improved. For this purpose, a histogram matching operation is performed, which increases the cleaning power of the algorithm between the vessel segment in the window and the background of the window. Radon conversion is then applied to image windows whose contrast is improved. Radon conversion is widely used in tomography or crop production (image generation of data projection associated with cross-sectional scans of an object). ${ }^{6-9}$ Shown as output shows the tomographic scan. Therefore, radon conversion inversion can be used to reconstruct the original density of the projection data. And thus form the mathematical basis for tomographic reconstruction, called image reconstruction. Radon conversion data is often referred to as a Sinogram because radon conversion is a Dirac delta function distributed on the graph of a sine wave. As a result, the radon transform has a number of graphical appearance objects such as several sinusoidal waves with different phases and amplitudes. Suppose $f(x)=f(x, y)$ is a fully supported continuous function on $R_{2}$. The radon $R f$ conversion will be equal to:

$$
\operatorname{Rf}(\mathrm{L})=\int_{\mathrm{L}} \mathrm{f}(\mathrm{x})|\mathrm{dx}|
$$

Parameterization of any straight line $\mathrm{L}$ with respect to the length of the arc $\mathrm{z}$ can always be written as follows:

$$
(x(z), y(z))=((z \sin \alpha+s \cos \alpha),(-z \cos \alpha+s \sin \alpha))
$$

Where $\mathrm{s}$ is the distance $\mathrm{L}$ from the origin and the angle that the normal vector $\mathrm{L}$ makes with the $\mathrm{x}$ axis. The values $(\mathrm{a}, \mathrm{s})$ can be considered as coordinates over the space of all lines in $\mathrm{R}_{2}$, and radon conversion can be expressed in these coordinates by the following equation:

$$
\operatorname{Rf}(\mathrm{a}, \mathrm{s})=\int_{-\infty}^{+\infty} \mathrm{f}(\mathrm{x}(\mathrm{z}), \mathrm{y}(\mathrm{z})) \mathrm{dz}=\int_{-\infty}^{+\infty} \mathrm{f}((\mathrm{z} \sin \alpha+\mathrm{s} \cos \alpha),(-\mathrm{z} \cos \alpha+\mathrm{s} \sin \alpha)) \mathrm{d} z
$$

As stated, radon conversion is based on integral absorption, and because the window diameter is longer than its side length, the maximum radon conversion is more likely to occur along the diameter. To solve this problem, first apply a circular mask to each window and then apply radon to it.

\section{Validation of the vessel with its refinement}

Verification of vessel segment detection in the process window is performed by checking the maximum amplitude value. Thus, the value of the maximum amplitude is compared with a threshold value and is confirmed if the detection accuracy is greater. If the vein segment is confirmed, the vein refinement stage will match the appearance of the vein fragment with the original vein fragment to obtain a good appearance and will be ignored if the candidate vein fragment is not approved. In order to obtain a proper representation of the diagnosed vessel fragment, first an approximate representation of the vessel is obtained with respect to the values and relationships of the preceding stage, and then by comparing this approximate representation with the original vessel fragment, an accurate representation of the vessel is obtained. To approximate the vein, the interval of the rods corresponding to the vessel segment is first determined. This interval revolves around the rod where the maximum is located. After calculating the interval above, a white square $m \times m$ is drawn on a black square, which is the approximate representation of the vein. To compare the approximate representation with the actual vessel segment and to obtain an accurate representation of the vessel segment, first the main window being processed is converted into a binary image where the white pixels represent the pixel-related pixels and the black pixels represent the pixels. Are related to the background. The threshold value used to binary the window is an average of two average values. Finally, by performing a logical AND between the binary window and the approximate representation, a precise representation of the fragment of the exposed vessel is obtained (Figure 3). ${ }^{10-15}$

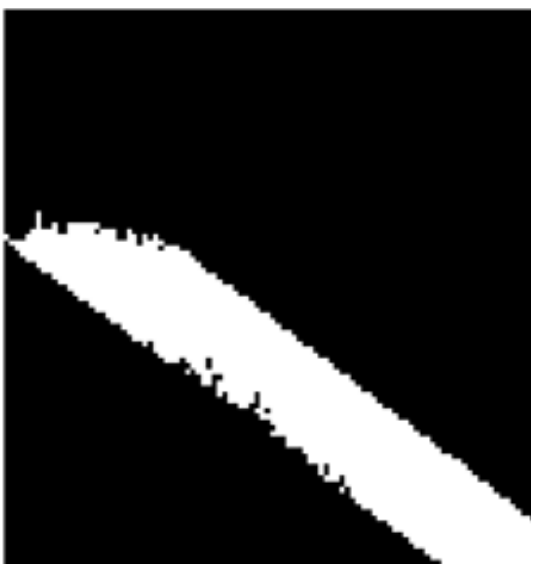

Figure 3 View the detailed image of the Vein.

\section{Conclusion}

To obtain the final representation of the retinal image veins, the fragments of veins revealed in different windows must be combined. Precise window displays are used to combine the results. So that two adjacent windows are overlapping, considering the overlap. In nonoverlapping areas, the end result is the exact window display, but in the overlapping areas, the results of two adjacent windows are OR consistent. After combining the results of all the windows, a binary image is obtained, the white pixels of which represent the arteries revealed by the retina image. Consequently, for each value of $t$, the final vascular representation was calculated for each of the 20 images and by comparing each result image with the corresponding detection result, both TPR and FPR rates were obtained for that image. The quantity of TPR is the ratio of the number of pixels in the result image correctly identified as a vessel to the total number of pixels in the result manual and the FPR is the ratio of the number of pixels in the result image that are mistakenly referred to as The veins are detected by the total number of non-vein pixels thus manually. Then the mean TPR and FPR values of all images were obtained for corresponding t. Due to the above process, it is now possible to diagnose Alzheimer's disease by counting blood vessels in the retina.

\section{Acknowledgments}

None.

\section{Conflicts of interest}

The authors declare there are no conflicts of interest.

\section{Funding}

None.

\section{References}

1. Du S, Huang D, Wang H. An adaptive support vector machine-based work piece surface classification system using high definition metrology. IEEE Trans Instrument Meas. 2015;64(10):2590-604. 
2. Mcbride JC, Zhao X, Munro NB, et al. Spectral and Complexity Analysis of Scalp EEG Characteristics for Mild Cognitive Impairmentand Early Alzheimer's Disease. Comput Methods Programs Biomed. 2014;114:153163.

3. Casson J, Yates DC, Smith SJ, et al. Wearable electroencephalography. IEEE Eng Med Biol Mag. 2010;29(3):44-56.

4. Kulkarni N, Bairagi V. Diagnosis of Alzheimer disease using EEG signals. Int J Eng Res Technol. 2014;3(4):1835-1858.

5. Haleh Aghajani, Edmond Zahedi, Mahdi Jalili, et al. Diagnosis of early Alzheimer's disease based on EEG source localization and a standardized realistic head model. IEEE journal of biomedical an dhealth informatics. 2013;17(6):1039-1045.

6. Ballard C, Gauthier S, Corbett A, et al. Alzheimer's disease. The Lancet. 2011;377(9770):1019-1031.

7. Gorji HT, Haddadnia J. A novel method for early diagnosis of Alzheimer's Disease based on pseudo Zernike moment from structural MRI. Neuroscience. 2015. p. 361-331.

8. Johnson KA, Fox NC, Sperling RA, et al. Brain imaging in Alzheimer disease. Cold Spring Harb Perspect Med. 2012;2(4):a006213.
9. Fowler MJ. Microvascular and Macrovascular Complications of Diabetes. Clinical Diabetes. 2008:26(2):77-82.

10. Staal J, Abramoff MD, Niemeijer M, etal. Ridge based vessel segmentation in color images of the retina. IEEE Trans Med Imaging. 2004;23(4):501509.

11. AlRawi M, Qutaishat M, Arrar M. An improved matched filter for blood vessel detection of digital retinal images.Comput Biol Med. 2007;37(2):262-267.

12. Estabridis K, Defigueiredo R. Blood Vessel Detection via [13].a Multiwindow Parameter Transform. Proceeding of the 19th IEEE Symposium on ComputerBased Medical Systems (CBMS'06). 2006 p. 22-23.

13. Dua S, Kandiraju N, Thompson H. Design and Implementation of a Unique Bloodvessel Detection Algorithm towards Eary Diagnoses of Diabetic Retinopathy. Proceeding of the 5th International Conference on Information Technology: Coding and Computing; 2005;1:26-31.

14. Teng T, Lefley M, Claremont D. Progress towards automated diabetic ocular screening: a review of image analysis and intelligent systems for diabetic retinopathy. Med Biol Eng Comput. 2002;40(1):2-13.

15. Matsuda H. MRI morphometry in Alzheimer's disease. Ageing Res Rev. 2016;30:17-24 